\title{
Análise do perfil epidemiológico da hanseníase em Maricá, Rio de Janeiro: uma contribuição da enfermagem
}

\author{
Analysis of the epidemiological profile of leprosy at Maricá, Rio de Janeiro: a contribution \\ from nursing
}

\section{Análisis del perfil epidemiológico de la lepra en Maricá, Rio de Janeiro: una contribuición de la enfermeria}

João Carlos Fialho de Oliveira'; Ana Maria Machado LeãoII; Fernanda Vasconcelos Spitz Britto ${ }^{\text {III }}$

\begin{abstract}
RESUMO: O objetivo da pesquisa foi analisar o perfil epidemiológico da hanseníase no município de Maricá, Rio de Janeiro. Estudo epidemiológico retrospectivo, com abordagem quantitativa. Utilizou dados do Sistema de Informação de Agravos de Notificação, de pacientes com hanseníase, no período entre 2000-2013. Incluiu as variáveis: número de casos notificados; idade; sexo; formas clínicas; classificação operacional; o grau de incapacidade dos acometidos e saída do sistema. Totalizaram 191 casos de hanseníase, sendo a maioria constituída de homens-54\%, maiores de 15 anos- $96 \%$, multibacilares- $63 \%$ e alta por cura como principal tipo de saída do sistema - 87,5\%. A taxa de detecção anual permaneceu média entre os maiores de 15 anos. Todos os examinados apresentaram algum grau de incapacidade física, durante o diagnóstico. Conclui-se que o conhecimento epidemiológico da doença na cidade implicará mais suporte para o controle de saúde pública local.

Palavras-Chave: Hanseníase; epidemiologia; enfermagem; saúde pública.
\end{abstract}

\begin{abstract}
The study aimed at analyzing epidemiological profile of leprosy in the city of Maricá, Rio de Janeiro, Brazil, from 2000 to 2013. This is a retrospective epidemiological study with quantitative approach. Data collected of all leprosy cases from Notifiable Diseases Information System Database through the notification of patients with leprosy. The variables included were: new cases diagnosed within the 2000-2013 span; age; clinic forms; operational classification; degree of physical disability and system output. 191 patients were reported: $54 \%$ males, $96 \%$ are older than 15 years old, $34 \%$ were in the virchowian leprosy, tuberculoid $(30 \%)$, dimorphic $(19 \%)$ and indeterminate $(13 \%), 63 \%$ multibacillary forms, $87.5 \%$ had had discharges after cure as main type of system output. Annual detection rate remained medium for those over 15 . All those examined showed physical disability to a certain extent at diagnosis time. We concluded that the epidemiological knowledge of leprosy in the city must require stronger support for disease control.
\end{abstract}

Keywords: Leprosy; epidemiology; nursing; public health.

RESUMEN: El objetivo del estudio fue analisar el perfil epidemiológico de la lepra en Maricá, Rio de Janeiro - Brasil. Estudio epidemiológico retrospectivo, con enfoque cantitativo. Fueron usados dados del Sistema de Información para Enfermedades de Declaración Obligatoria, a través de la notificación de los pacientes con lepra, de 2000 a 2013. Las variables incluídas fueron: número de casos notificados; edad; sexo; formas clínicas; clasificación operacional y el grado de discapacidad de los afectados y salida del sistema. Totalizaron 191 casos, con prevalencia de 54\% de hombres, 96\% mayores de 15 años, 63\% de multibacilares y $87,5 \%$ de curación como el principal tipo de salida. La tasa de detección anual se mantuvo media para los > de 15 años. Todos los pacientes presentaron alguno grado de discapacidad en el momento del diagnóstico. Se concluye que el conocimiento epidemiológico de la enfermadad en la ciudad significará más apoyo para el control de la salud.

Palabras Clave: Lepra; epidemiología; enfermería; salud pública.

\section{INTRODUÇÃO}

A hanseníase é conhecida desde os tempos bíblicos:

E aconteceu que, quando estava numa daquelas cidades, eis que um homem cheio de lepra, vendo a Jesus, prostrou-se sobre o rosto, e rogou-lhe, dizendo: Senhor, se quiseres, bem podes limparme. E ele, estendendo a mão, tocou-lhe, dizendo: Quero, sê limpo. E logo a lepra desapareceu dele ${ }^{1: 72}$.

Naquela época, a hanseníase era chamada de lepra. As mutilações ocasionadas e o desconhecimento geraram medo, atitudes preconceituosas de rejeição e discriminação de seu portador. A história da lepra é conhecida e perpassa até os dias atuais no imaginário de muitas pessoas contribuindo para o estigma e outras repercussões desfavoráveis. Por isso, em 1995 o governo brasileiro determinou a mudança para o termo hanseníase mediante a Lei 9.0102,3.

É uma doença infectocontagiosa, crônica causada pela bactéria Mycobacterium leprae, tem a capaci-

'Graduado em Enfermagem pela Faculdade de Enfermagem da Universidade do Estado do Rio de Janeiro. Brasil. E-mail: jo_ca_joca@hotmail.com IIMestre em Enfermagem. Professora Assistente do Departamento de Enfermagem em Saúde Pública da Faculdade de Enfermagem da Universidade do Estado do Rio de Janeiro.Brasil. E-mail: ammleao@gmail.com

IIIMestre em Enfermagem. Secretária Municipal de Saúde de Maricá. Rio de Janeiro, Brasil. E-mail: fespitz@gmail.com

Iv Trabalho premiado em 1ㅇ lugar no ENFCUIDAR. Faculdade de Enfermagem da Universidade do Estado do Rio de Janeiro. Brasil, 2014. 
dade de infectar grande número de indivíduos, porém, poucos adoecem. Portanto, o domicílio é apontado como importante espaço de transmissão da doença ${ }^{2,4}$.

De acordo com o boletim epidemiológico da Organização Mundial da Saúde (OMS), a taxa de detecção nas Américas foi de 4,18 casos por 100.000 habitantes durante o ano de 2011. Nestas regiões os dados foram fortemente influenciados pelo Brasil com 33.955 casos - o segundo país em número de casos no mundo - sendo 16\% de novos casos (em 2011) registrado no território brasileiro, depois da Índia (58\%) e seguido pela Indonésia $(9 \%)^{5}$.

No Brasil, acontece alta deteç̧ão em alguns estados do Norte, Nordeste e Centro-Oeste, mas a tendência da detecção de casos novos de hanseníase é decrescente no país. Além das diferenças epidemiológicas, as informações apresentadas permitem visualizar problemas operacionais que revelam a necessidade de maior empenho dos estados, na implementação das ações estratégicas previstas no Pacto pela Vida, Programa Ambientes Verdes e Saudáveis e Programa de Aceleração do Crescimento (PAC) - Mais Saúde para melhoria da atenção integral à pessoa com hanseníase ou com sequelas da doença ${ }^{6}$.

A meta de eliminação da hanseníase, com base no indicador de prevalência pontual, foi substituída pelo indicador de detecção de casos novos. A Região Sudeste e o Estado do Rio de Janeiro apresentam tendência decrescente, estatisticamente significativa, de coeficientes de detecção. A região metropolitana do Rio de Janeiro concentra $80 \%$ dos casos novos, sugerindo uma relação entre altas taxas de detecção com maior concentração populacional e com maior número de unidades de saúde descentralizadas ${ }^{6,7}$.

Espera-se que este estudo possa contribuir para a orientação dos profissionais, em especial os enfermeiros que atuam com planejamento e gestão de políticas de saúde e, portanto, com ampliação do acesso e do cuidado. Desse modo, o objeto deste estudo é o levantamento da situação da hanseníase no município de Maricá, do ano 2000 a 2013, objetivando-se analisar o perfil epidemiológico da hanseníase no município de Maricá, nesses 14 anos.

O município escolhido deveu-se pela experiência profissional de uma das autoras deste trabalho e sua atuação no programa de hanseníase. A escolha da delimitação temporal de 2000 a 2013, se justifica devido aos programas lançados pelo Ministério da Saúde (MS) que tiveram como meta a eliminação da doença.

A meta anterior, estabelecida pela OMS, para a eliminação da hanseníase como problema de saúde pública foi a redução de sua prevalência para menos de 1 caso em 10.000 habitantes até o final de 2005, e, posteriormente, até 2010, critério este não alcançado pelo Brasil ${ }^{8}$. Por isso, houve ampliação do prazo, até $2015^{9}$.

\section{REVISÃo DE LITERATURA}

O MS divulgou, em 2002, orientações para os profissionais de saúde com o objetivo de aperfeiçoar as medidas voltadas para a integração e a efetividade das ações de controle da doença, na rede básica de saúde, como: fazer o diagnóstico diferencial de caso de hanseníase; entender seus aspectos epidemiológicos relacionados ao agente etiológico e ao modo de transmissão; seus aspectos clínicos como, sinais e sintomas dermatológicos e neurológicos. Contemplam também os mais importantes e atualizados conhecimentos para a abordagem do paciente, configurando, portanto, instrumento relevante para o atendimento adequado e resolutivo ${ }^{2}$.

Nesse contexto, o controle da hanseníase no Brasil exige parcerias, grande mobilização social, vontade política dos gestores, compromisso e motivação dos técnicos e controle social ${ }^{6}$. Sobretudo, por ser uma doença com agravantes inerentes às patologias de origem socioeconômica e cultural, é também marcada pela repercussão psicológica gerada pelas possíveis deformidades e incapacidades físicas decorrentes do processo de adoecimento. São essas deformidades e incapacidades físicas uma das causas do estigma e do isolamento da pessoa na sociedade ${ }^{10}$.

Entre os profissionais de saúde, o enfermeiro possui um papel fundamental no controle da patologia, pois ele está em contato direto com a comunidade, nos programas de atenção primária e nos centros de atendimento aos portadores da hanseníase. Portanto, facilitando o tratamento da mesma e tendo como principal finalidade a melhoria da qualidade de vida dos acometidos ${ }^{11}$.

\section{Metodologia}

Foi estudada a população do município de Maricá, no Estado do Rio de Janeiro, estimada em 2013 de 139.552 habitantes, numa área territorial de 362,57 $\mathrm{km}^{2}$, com densidade demográfica de $351,55 \mathrm{hab} / \mathrm{km}^{2}{ }^{12}$.

Trata-se de um estudo epidemiológico retrospectivo com abordagem quantitativa e analítica, realizado com dados do Sistema de Informação de Agravos de Notificação (SINAN) para identificar o perfil epidemiológico da hanseníase em Maricá, no período de 2000 a 2013.

Os critérios de inclusão foram as notificações de pacientes com diagnóstico de hanseníase, na cidade de Maricá, registradas no SINAN, no período escolhido. Os critérios de exclusão foram as notificações duvidosas, incompletas, com registro de outro período e as variáveis não selecionadas para este estudo.

Utilizou-se como fonte de informação o SINAN, no que se refere aos dados da Secretaria Municipal de Saúde de Maricá (SMSM). Para atender ao objetivo do estudo foram selecionadas as variáveis: número de 
casos notificados de 2000 a 2013; faixa etária (menores e maiores de 15 anos); sexo; formas clínicas da doença baseada na classificação de Madri de $1953^{13}$; classificação operacional paucibacilar (PB) ou multibacilar (MB); grau de incapacidade física (GIF); e o tipo de saída do sistema.

A amostra do estudo correspondeu às pessoas que adoeceram de hanseníase no período selecionado. A coleta de dados foi realizada por etapas, incluídas as variáveis mostradas no SINAN, que foram incorporados às planilha do Microsoft Excel 2007. Para a análise estatística, os dados foram apresentados em frequência absoluta e relativa percentual, com auxílio de tabelas.

Baseado na Portaria $n^{\circ} 3.125$, de 7 de outubro de 2010, que aprova as diretrizes para vigilância, atenção e controle da hanseníase ${ }^{14}$, foi estimado o perfil epidemiológico.

O SINAN é alimentado pela notificação e investigação dos casos de patologias e agravos, que constam da lista nacional de doenças de notificação compulsória, inclusive a hanseníase, onde cada caso diagnosticado deve ser informado na semana epidemiológica de ocorrência, pelos profissionais da vigilância, utilizando-se ficha específica, nos três níveis de atenção à saúde ${ }^{14}$.

Este estudo constitui-se em uma pesquisa documental e eletrônica, portanto, não houve necessidade de aprovação pelo Comitê de Ética em Pesquisa. Foi solicitada autorização à SMSM, através do ofício n 624/2014, para a coleta dos dados do município no SINAN.

Após aprovação/aceite do ofício, iniciou-se a coleta dos dados no referido sistema, que aconteceu em maio de 2014.

\section{Resultados e Discussão}

De 2000 a 2013, um total de 191 pacientes de Maricá foi notificado no SINAN. A taxa de detecção de 2000 a 2009 não foi calculada devido à falta de registro da população pelo Instituto Brasileiro de Geografia e Estatística (IBGE). Sabe-se que, no período de 2010 a 2013, a classificação da taxa de detecção oscilou entre média de 2 a 9,99 casos/100.000 hab. e alta de 10 a 19,99 casos/100.000 hab., conforme a Tabela 1.

TABELA 1: Taxa de detecção anual de novos casos de hanseníase na população, Maricá-RJ, 2010-2013.

\begin{tabular}{cccc}
\hline Ano & $\begin{array}{c}\text { Número de } \\
\text { casos }\end{array}$ & $\begin{array}{c}\text { Taxas de } \\
\text { detecção }\end{array}$ & Resultado \\
\hline 2010 & 11 & 8,6 & MÉDIA \\
2011 & 11 & 8,4 & MÉDIA \\
2012 & 14 & 10,4 & ALTA \\
2013 & 9 & 6,4 & MÉDIA \\
\hline
\end{tabular}

Fonte: SINAN
A análise da distribuição da idade dos casos justificados revela que a maioria - $183(96 \%)$ - tinha 15 anos ou mais e que 8 (4\%) casos investigados se situavam abaixo de 15 anos. A relação entre faixa etária e sexo mostrou o adoecimento maior em homens de 60 a 69 anos e mulheres, de 20 a 29 anos e 30 a 39. Estudo mostra que a hanseníase acometeu pessoas de faixa etárias entre 30 a 49 anos predominantemente ${ }^{15}$. Tais resultados refletem em parte o perfil etário da população desta pesquisa.

O coeficiente, em menores de 15 anos de idade, alcançou um nível alto (5,0 a 9,99/100.000 hab.) nos 14 anos avaliados neste estudo. Indica a possibilidade de expansão da doença e da intensidade de transmissão recente da endemia ${ }^{14}$. Esse dado é importante, pois a redução de casos em menores de 15 anos é prioridade do Plano Nacional de Controle da Hanseníase, sendo o indicador de eliminação da hanseníase no PAC - Mais Saúde ${ }^{6}$.

presente trabalho se assemelha a outros que mostraram o acometimento da hanseníase em pessoas de mais idade. Esta alta frequência em adultos se deve ao longo período de incubação do bacilo, que varia de 2 a 7 anos. Além disso, a hanseníase acomete pessoas economicamente ativas, e com isso traz perdas econômicas e sociais, interfere na capacidade de realização de atividades diárias dos indivíduos acometidos, independente do GIF que apresentam ${ }^{15-17}$.

grande aumento de notificações em Maricá, em 2005, sugere ser resultado da campanha publicitária de grande porte que o governo lançou, em 2004, em nível nacional, visando o combate à hanseníase no país, inclusive para a sua eliminação no Brasil até o final de 2005.

Para o aumento das notificações, no período de 2000 a 2013, indicam-se como justificativas a execução das políticas de controle, a organização do sistema de saúde e a notificação compulsória da hanseníase, que se estruturaram em todo o território nacional, através da investigação obrigatória em todos os níveis de atenção à saúde. Os casos diagnosticados devem ser oficialmente comunicados, utilizando-se a ficha específica de notificação e investigação do SINAN ${ }^{14}$. Outros fatores influenciam como a existência real de casos transmissores e da agilidade diagnóstica no sistema de saúde, as alterações operacionais do sistema de vigilância epidemiológica, algumas conhecidas outras desconhecidas e sua descentralização do sistema de atenção à saúde ${ }^{18}$.

As variações observadas de casos foram causadas principalmente pelas oscilações da transmissão da hanseníase e mudanças operacionais do programa ${ }^{19}$. Paradoxalmente, a tendência de diminuição de casos acontece no Brasil - especialmente em Maricá, à medida que os diagnósticos e o tratamento dos doentes foram realizados, mediante as estratégias de descentralização do atendimento, amparadas pelos protocolos do $\mathrm{MS}^{19}$. 
Quanto à análise do sexo, a maioria - 103(53,6\%) - dos casos foi registrada em homens, exceto em 2001, 2010 e 2012, quando a maioria foi representada por mulheres com $9(64,2 \%), 6(54,5 \%)$ e $11(73,3 \%)$ casos, respectivamente, segundo a Tabela 2 .

Igualmente em outro estudo, realizado no Maranhão, a maioria dos casos incidiu sobre o sexo masculino. Isso indica que o homem é o mais afetado. No entanto, no mesmo estudo, relata-se um aumento no número de casos em mulheres ${ }^{16}$.

Considerando as formas clínicas, houve a predominância da forma virchowiana em 34,2\% dos casos, seguidos pela tuberculoide $(30 \%)$, indeterminada $(13,4 \%)$, dimorfa $(18,7 \%)$ e formas não classificadas $(3,7 \%)$, como expõe a Tabela 2 . Este estudo constatou uma incidência maior de casos multibacilares (MB paciente portador de mais de cinco lesões na pele). Eles têm maior risco de transmissão, enquanto as formas paucibacilares $(\mathrm{PB}$ - pacientes portadores de até cinco lesões) têm baixo risco de transmitir para os indivíduos que estejam em contato próximo ${ }^{20}$.

Outras pesquisas também mostraram resultados semelhantes ${ }^{17}$, embora outros estudos afirmem que a forma PB não é transmissível devido à baixa produção de bacilos $^{21}$. À medida que se obtém mais casos MB, pode-se pensar em aumento da endemia, quando os indivíduos mais suscetíveis são afetados e também com um sistema de atendimento primário e epidemiológico ineficiente que permite a perpetuação do foco de transmissão ${ }^{22}$.

Paralelamente, grupos de pacientes estudados com hanseníase PB mostraram pouco comprometimento da qualidade de vida. Isto leva a concluir que, quanto mais precoce é o diagnóstico e o tratamento, menor é o acometimento da qualidade de vida ${ }^{23}$.

Existe discordância no somatório de MB (formas virchowiana e dimorfa) e nos PB (tuberculoide e indeterminada), pois 180 pessoas adoeceram segundo as formas clínicas. Isso pode ter ocorrido devido às perdas de algumas informações ao longo das atualizações de sistema para notificação. Vale mencionar que o total de $\mathrm{PB}$ registrado destaca 71 pacientes, com isso, a soma de 25 casos - forma indeterminada - com os 56 casos - forma tuberculoide - registrados foi discordante; isto também ocorreu na soma total de 64 casos na forma virchowiana e 35 casos na forma dimorfa, sendo 99 pacientes MB, provavelmente devido a erros na classificação ou registro.

TABELA 2: Perfil dos pacientes diagnosticados e notificados no Sistema de Informação de Agravos de Notificações, Maricá-RJ, 2000-2013.

\begin{tabular}{|c|c|c|c|c|c|c|c|c|c|c|c|c|c|c|c|c|}
\hline \multirow[t]{2}{*}{ Variáveis } & 2000 & 2001 & 2002 & 2003 & 2004 & 2005 & 2006 & 2007 & 2008 & 2009 & 2010 & 2011 & 2012 & 2013 & To & tal \\
\hline & $\mathrm{f}$ & $\mathrm{f}$ & $f$ & $\mathrm{f}$ & $f$ & $f$ & $f$ & $f$ & $\mathrm{f}$ & $\mathrm{f}$ & $f$ & $\mathrm{f}$ & $f$ & $\mathrm{f}$ & $f$ & $\%$ \\
\hline \multicolumn{17}{|l|}{ Sexo } \\
\hline Masculino & - & 5 & 9 & 12 & 8 & 19 & 12 & 2 & 7 & 7 & 5 & 7 & 4 & 6 & 103 & 53,60 \\
\hline Feminino & - & 9 & 5 & 10 & 8 & 15 & 10 & 2 & 2 & 3 & 6 & 5 & 11 & 3 & 89 & 46,00 \\
\hline Subtotal & - & 14 & 14 & 22 & 16 & 34 & 22 & 4 & 9 & 10 & 11 & 12 & 15 & 9 & 192 & 100 \\
\hline \multicolumn{17}{|l|}{ Classificação } \\
\hline \multicolumn{17}{|l|}{ Operacional } \\
\hline Paucibacilar & - & 5 & 2 & 6 & 5 & 10 & 9 & 4 & 5 & 5 & 5 & 6 & 6 & 3 & 71 & 36,97 \\
\hline Multibacilar & 2 & 8 & 11 & 15 & 12 & 24 & 13 & 1 & 3 & 5 & 6 & 5 & 9 & 6 & 120 & 62,51 \\
\hline Ignorado/Branco & - & - & - & 1 & - & - & - & - & - & - & - & - & - & - & 1 & 0,52 \\
\hline Subtotal & 2 & 13 & 13 & 22 & 17 & 34 & 22 & 5 & 8 & 10 & 11 & 11 & 15 & 9 & 192 & 100 \\
\hline \multicolumn{17}{|l|}{ Forma clínica } \\
\hline Indeterminada & - & 4 & 2 & 1 & 2 & 7 & 2 & 1 & - & 1 & 2 & - & 2 & 1 & 25 & 13,40 \\
\hline Tuberculoide & - & 3 & 2 & 8 & 4 & 7 & 7 & 2 & 5 & 3 & 3 & 6 & 4 & 2 & 56 & 30,00 \\
\hline Dimorfa & - & 1 & 2 & 6 & 3 & 11 & 6 & - & 1 & - & 1 & 1 & 1 & 2 & 35 & 18,70 \\
\hline Virchowiana & 2 & 4 & 7 & 7 & 7 & 9 & 7 & - & 2 & 3 & 3 & 3 & 8 & 2 & 64 & 34,20 \\
\hline Não classificado & - & - & - & - & 1 & - & - & - & - & 1 & 2 & 1 & - & 2 & 7 & 3,70 \\
\hline Subtotal & 2 & 12 & 13 & 22 & 17 & 34 & 22 & 3 & 8 & 8 & 11 & 11 & 15 & 9 & 187 & 100 \\
\hline \multicolumn{17}{|l|}{ Tipo de saída } \\
\hline Cura & 2 & 12 & 12 & 20 & 16 & 31 & 18 & 4 & 7 & 9 & 9 & 10 & 10 & 1 & 161 & 87,50 \\
\hline Óbito & - & - & - & 2 & - & - & 1 & - & 1 & 1 & - & 1 & 1 & - & 7 & 3,80 \\
\hline Abandono & - & - & - & - & 1 & 1 & 1 & - & - & - & 2 & - & 2 & - & 7 & 3,80 \\
\hline Erro diagnóstico & - & - & - & - & - & - & 1 & 1 & - & - & - & - & 1 & - & 3 & 1,63 \\
\hline TNE & - & - & 1 & - & - & 1 & 1 & - & - & - & - & - & - & - & 3 & 1,63 \\
\hline TM & - & 1 & - & - & - & - & - & - & - & - & - & - & 1 & - & 2 & 1,09 \\
\hline $\mathrm{TE}$ & - & - & - & - & - & 1 & - & - & - & - & - & - & - & - & 1 & 0,54 \\
\hline Subtotal & 2 & 13 & 13 & 22 & 17 & 34 & 22 & 5 & 8 & 10 & 11 & 11 & 15 & 1 & 184 & 100 \\
\hline
\end{tabular}

Fonte: SINAN

Legenda: TNE-Transferência não especificada; TM-transferência de município; TE-transferência de estado 
Assim, na população pesquisada, a forma $\mathrm{MB}$ foi predominante, $62,5 \%$. Houve erro nos registros e os valores não conferem com as formas clínicas. A partir dessa realidade - da dificuldade de confirmar as formas clínicas, conforme o estabelecido pela classificação de Madri $1^{13}$ - a classificação operacional do MS (em PB e $M B)^{2}$ veio simplificar e facilitar esse procedimento a fim de efetivar o tratamento do doente, pois o mesmo só iniciará o tratamento com a definição do caso.

Observando as formas clínicas na população, houve um aumento de 2000 a 2005 e um significativo decréscimo, a partir de 2006, com uma tendência a estabilização. A forma clínica predominante foi a virchowiana com 64(34\%) casos que foram somados aos $120(62,5 \%)$ casos MB encontrados. O somatório da forma virchowiana e dimorfa leva ao predomínio das formas contagiantes, identificadas tardiamente. Assim, pode-se falar em transmissores do bacilo sem diagnóstico precoce, sem avaliação profissional capacitada para fazer um exame físico completo. Entretanto, de 2007 a 2011, pode-se observar mais formas PB (devido à forma tuberculoide) do que $\mathrm{MB}$, sendo que a tuberculoide acomete indivíduos resistentes, enquanto que a dimorfa e a virchoviana implicam a perpetuação da transmissão, com expressivo poder incapacitante ${ }^{24}$.

Foram examinados $157(85,8 \%)$ dos 183 pacientes registrados e $51(27,9 \%)$ apresentaram algum GIF(1 ou 2). Não ocorreram casos com GIF 2 nos anos 2000 a 2002, 2007 e 2008; e a proporção de casos de hanseníase com GIF 2, no momento do diagnóstico, foi considerado médio (5 a 9.9\%) para o período de 2004 a 2006; de 2009 a 2013 foi alto (maior ou igual a $10 \%)$. Neste estudo, foram $157(85,8 \%)$ pacientes avaliados para GIF, em todos os anos pesquisados, tendo sido registrado que todos eles apresentaram algum GIF (1 ou 2). O contingente avaliado de 85,8\% com incapacidade física é considerado insatisfatório, sendo menor do que a quantidade avaliada no Estado do Rio de Janeiro de 2001 a 2008, onde alcançou $88,4 \%$, no período citado ${ }^{6}$.

Somaram 106(67,5\%) pacientes sem incapacidade física (grau - zero) e $33(21 \%)$ com GIF1 e 18(11,46\%) com GIF2, em Maricá. O predomínio do GIF zero é informado em outros estudos ${ }^{22,25}$. As porcentagens de casos de pacientes com GIF2, no momento do diagnóstico, foram baixas em 2003 e, depois, predominando média e alta, corroborando os achados do Estado do Rio de Janeiro de 2001 a 2008 ${ }^{6}$. No Brasil, dos casos novos, 23,3\% estão relacionados com GIF1 e GIF2 ${ }^{10}$.

Relacionado com o tempo de doença, o GIF, é um indicador que permite uma avaliação indireta da efetividade das atividades de detecção precoce e tratamento adequado dos $\operatorname{casos}^{26}$.

O predomínio das formas $\mathrm{MB}$ e com porcentagem baixa de GIF 2 demonstram o atraso no diagnóstico na área, consequentemente, evidências permitem supor o despreparo dos profissionais para o reconhecimento das complicações dessa micobacteriose, podendo-se questionar o controle da epidemiologia da doença na localidade ${ }^{27}$.

O GIF é a avaliação que indica a existência de perda da sensibilidade protetora e/ou deformidades visíveis em consequência de lesão neural ${ }^{10}$.

O GIF2, refere: nos olhos, compreende a presença de algum sinal e/ou sintoma como o lagoftalmo e/ou ectrópio, triquíase, opacidade corneana central e até cegueira; nas mãos, pode revelar lesões tróficas e/ou lesões traumáticas, garras, reabsorção e mão caída; e nos pés, podem existir lesões tróficas e/ou lesões traumáticas, garras, reabsorção, pé caído e contratura do tornozelo ${ }^{10: 109}$.

Essas lesões são decorrentes de afecções dos nervos periféricos e suas fibras sensitivas, motoras e autonômicas, sendo o nervo facial (VII par craniano), nervo trigêmeo ( $\mathrm{V}$ par craniano), nervo ulnar, nervo mediano, nervo radial, nervo fibular comum e nervo tibial envolvidos com maior frequência ${ }^{10}$.

No período estudado, foram notificados 184 casos com registro sobre o tipo de saída dos pacientes no sistema de informação,segundo o ano de diagnóstico. Foram $161(87,5 \%)$ que receberam alta por cura, ocorreram $7(3,8 \%)$ casos de óbito e abandono, 3(1,63\%) tiveram erro de diagnóstico, 3(1,63) transferências não especificadas, $2(1,09 \%)$ transferências para outro município e $1(0,54 \%)$ transferência para outra unidade federativa. Vale destacar que a maioria dos pacientes cadastrados teve a cura da doença, por ter concluído o tratamento.

A saída por abandono deverá ser informada quando o doente que ainda não concluiu o tratamento não compareceu ao serviço de saúde nos últimos 12 meses, tanto nos casos PB quanto nos $\mathrm{MB}^{14}$. Situação não desejada, confirma o desconhecimento da situação do paciente, pela unidade de saúde.

Esses achados mostram que o desfecho da doença é interessante: grande parte dos pacientes se comprometeu com o tratamento e aceitou o uso da poliquimioterapia (PQT) como ferramenta para alcançar a cura da doença. Vale ressaltar que a cura e o tratamento da pessoa são demorados, através da administração dos esquemas de tratamento PQT com obediência aos prazos estabelecidos: de 6 a 9 meses para os casos $\mathrm{PB}$ e de 12 a 18 meses para os casos $\mathrm{MB}^{2}$.

Dados de outro estudo sugerem que os casos de abandono são devidos à fragilidade na credibilidade por parte do paciente, do diagnóstico médico e a não aceitação do uso de PQT, necessitando que a atenção dada por parte dos serviços de saúde seja enfatizada na orientação dos portadores, despertando neles a consciência de que a adesão ao tratamento de forma objetiva e continuada o levará à obtenção da cura da enfermidade ${ }^{28}$. 
Como o adoecer de hanseníase abrange o desconhecimento ou idéias antigas e preconceituosas relacionadas com questões sociais, culturais e emocionais e devido às características da doença, os profissionais de enfermagem, ao receberem pacientes com hanseníase devem atentar para as questões étio-legais relativas ao preconceito e a discriminação em suas consultas. Além disso, os enfermeiros podem contribuir com as comunidades, auxiliando na redução da disseminação da doença e no aumento da procura de diagnóstico precoce; trabalhar com seus conhecimentos e vivências, favorecendo o enfrentamento de seus próprios estigmas; bem como auxiliar no enfrentamento positivo desses indivíduos frente à doença, fortalecendo os fatores protetores, buscando a detecção de fatores de risco pelo trabalho conjunto com a família e redes de apoio e orientando acerca do autocuidado para prevenir incapacidades e manter a autoimagem positiva ${ }^{29-32}$.

\section{ConClusão}

O MS tem o compromisso de eliminação da hanseníase até 2015, ou seja, alcançar menos de 1 caso por 10.000 habitantes. Este estudo verificou que, no município de Maricá, a magnitude da morbidade teve parâmetro médio (6,4 casos/100.000 habitantes) em 2013. Assim, essa cidade está no passo certo no controle da doença, seguindo o plano integrado de ações estratégicas do governo, já que ocorreu a diminuição do número de casos diagnosticados e um amplo número de altas.

O coeficiente de detecção - indicador de transmissibilidade da hanseníase - diminuiu durante os últimos quatro anos do estudo. Mesmo assim, aponta que ainda existe circulação de bacilos no município.

O sexo masculino e as pessoas acima de 15 anos foram os mais acometidos. Houve maior número de notificações da forma clínica virchowiana, contribuindo para a maioria de casos $\mathrm{MB}$ e o GIF -2 no diagnóstico. Indica a demora na detecção e no início do tratamento e possíveis implicações psicológicas, perpetuação do estigma e contínua transmissão dos bacilos pelas pessoas doentes.

Contudo, é importante ressaltar o expressivo quantitativo que concluiu o tratamento, correspondendo à alta por cura, na saída do sistema. A maioria confiou no tratamento, na medicação e na atuação dos profissionais da unidade de saúde, pois poucos pacientes abandonaram o tratamento.

Vale ressaltar a necessidade de intensificação e fortalecimento da vigilância em hanseníase, pois ela subsidia recomendações, a promoção e a análise da efetividade das intervenções, determinando que os registros dos casos diagnosticados e sob tratamento sejam atualizados continuamente. É essencial a divulgação das informações obtidas como fonte de planejamento e avaliação das gerências a serem desencadeadas.
Este estudo encontrou algumas limitações como ausência de preenchimento de certos dados na ficha de notificação do SINAN, dificultando uma avaliação diagnóstica completa e fiel à realidade do município. Outro fato é que toda informação coletada é secundária e depende da entrada de dados no sistema de informação por um profissional, que nem sempre é da área da saúde.

Esta pesquisa contribui para a orientação dos profissionais, em especial dos enfermeiros que atuam em planejamento e gestão de políticas de saúde e em prevenção e controle de doenças transmissíveis.

\section{REFERÊNCIAS}

1.Bíblia Sagrada. Antigo e novo testamento. Traduzido em português por João Ferreira de Almeida. 31aㅡ ed. Rio de Janeiro: Editora Contexto; 1990.

2.Ministério da Saúde (Br). Secretaria de Políticas de Saúde. Departamento de Atenção Básica. Guia para o Controle da hanseníase. 3ª ed. Brasília (DF): Ministério da Saúde; 2002.

3.Governo Federal (Br). Lei 9.010 de 29 de março de 1995. Dispõe sobre a terminologia oficial relativa à hanseníase e dá outras providências. [citado em 15 jun 2014] Disponível em: http://www.planalto.gov.br/ ccivil 03/leis/L9010.htm.

4.Opromolla PA, Laurenti R. Controle da hanseníase no Estado de São Paulo: análise histórica. Rev Saúde Pública. 2011; 45(1):196.

5.Organização Mundial da Saúde. Situação global da hanseníase. Boletim epidemiológico semanal. [Internet]. 2012 ago [citado em 01 fev 2014]; 87:317-28. Disponível em: http://www.who.int/wer/2012/wer8734.pdf?ua=1. 6.Ministério da Saúde (Br). Secretaria de Vigilância em Saúde. Hanseníase no Brasil dados e indicadores selecionados. Brasília (DF): Ministério da Saúde; 2009. 7.Valle CLP. Situação da hanseníase no Estado do Rio de Janeiro no período de 2001 a 2009. Rev Hospital Universitário Pedro Ernesto. 2011;10(1):11-8.

8.Secretaria de Estado de Saúde do Distrito Federal (Br). Hanseníase: Protocolo de Atendimento. Brasília (DF): Subsecretaria de Vigilância à Saúde; 2007.

9.Ministério da Saúde (Br). Secretaria de Vigilância em Saúde.Plano integrado de ações estratégicas de eliminação da hanseníase ... Plano de ação 2011-2015. Brasília (DF): Editora MS; 2012.

10.Ministério da Saúde (Br). Secretaria de Vigilância em Saúde. Manual de prevenção de incapacidades. Cadernos de prevenção e reabilitação em hanseníase. Brasília (DF): Editora MS; 2008.

11.Silva JMM, Moreira MPS, Silva TMV. O papel do enfermeiro no tratamento da hanseníase. Revista Universo da Enfermagem. 2014; 3(1):57-61.

12.Instituto Brasileiro de Geografia e Estatística. Assistência Médica Sanitária 2009 [Internet]. Rio de Janeiro: IBGE; 2010 [citado em 08 mai 2014]. Disponível em: http://cidades.ibge.gov.br/xtras/perfil. php? codmun $=330270$. 
13.Montenegro RMN, Zandonade E, Molina MDC, Diniz LM. Reactional state and nutritional profile among leprosy patients in the primary health care system, Greater Vitória, Espírito Santo State, Brazil. Cad Saúde Pública. 2012; 28:31-8.

14.ANVISA (Br). Portaria no 3.125, de 7 de outubro de 2010. Aprova as Diretrizes para Vigilância, Atenção e Controle da hanseníase. [citado em 02 jun 2014]. Disponível em: http://www.anvisa.gov.br/hotsite/talidomida/ legis/portaria n 3125 hanseniase 2010.pdf.

15.Duarte MTC, Ayres JA, Simonett IJP. Perfil socioeconômico e demográfico de portadores de hanseníase atendidos em consulta de enfermagem. Rev Latino-Am Enfermagem [online]. 2007; 15 (spe): 774-9.

16.Corrêa RGCF, Aquino DMC, Caldas AJ, Amaral DKCR, França FS, Mesquita, ERRBPL. Epidemiological, clinical, and operational aspects of leprosy patients assisted at a referral service in

the state of Maranhão, Brazil. Revista da Sociedade Brasileira de Medicina Tropical. 2012; 45(1):89-94.

17. Batista AMN. Avaliação da incapacidade e limitação de atividades em Pacientes afetados pela hanseníase: uma análise do escore Salsa [monografia]. Bauru (SP): Secretaria de Estado da Saúde; 2010.

18.Penna MLF, Oliveira MLW, Carmo EH, Penna GO, Temporão JG. Influência do aumento do acesso à at- enção básica no comportamento da taxa de detecção de hanseníase de 1980 a 2006. Revista da Sociedade Brasileira de Medicina Tropical. 2008; 41 (supl 2):6-10. 19.Silva Sobrinho RA, Mathias TAF. Perspectivas de eliminação da hanseníase como problema de saúde pública no Estado do Paraná, Brasil. Cad Saúde Pública. 2008; 24:303-14.

20.Murto C, Ariza L, Alencar CH, Chichava AO, Oliveira AR, Kaplan C, Silva LF, Heukelbach J.C Migration among individuals with leprosy: a population-based study in Central Brazil. Cad Saúde Pública. 2014; 30:487-501. 21.Macedo CP, Cerqueira MFF, Poveda VB. Avaliação do perfil epidemiológico da hanseníase em um município do interior paulista nos anos de 2000 a 2006. Revista REENVAP. 2014; 4:13

22.Melão S, Blanco LFO, Mounzer N, Veronezi CCD, Simões PWTA. Perfil epidemiológico dos pacientes com hanseníase no extremo sul de Santa Catarina, no período de 2001 a 2007. Revista da Sociedade Brasileira de Medicina Tropical. 2011; 44:79-84

23.Bottene IMC, Reis VMS. Quality of life of patients with paucibacillary leprosy. An Bras Dermatol. 2012; $87: 408-11$

24.Crespo MJI, Gonçalves A, Padovani CR. Hanseníase: pauci e multibacilares estão sendo diferentes? Medicina (Ribeirão Preto). 2014; 47:43-50. 